University of South Carolina

Scholar Commons

2010

\title{
An Efficient Electrochemical-Thermal Model for a Lithium-Ion Cell by Using the Proper Orthogonal Decomposition Method
}

Long Cai

Ralph E. White

University of South Carolina - Columbia, white@cec.sc.edu

Follow this and additional works at: https://scholarcommons.sc.edu/eche_facpub

Part of the Transport Phenomena Commons

\section{Publication Info}

Published in Journal of the Electrochemical Society, Volume 157, Issue 11, 2010, pages A1188-A1195.

(c) The Electrochemical Society, Inc. 2010. All rights reserved. Except as provided under U.S. copyright law, this work may not be reproduced, resold, distributed, or modified without the express permission of The Electrochemical Society (ECS). The archival version of this work was published in

Cai, L, \& White, R.E. (2010). An Efficient Electrochemical-Thermal Model for a Lithium-lon Cell by Using the Proper Orthogonal Decomposition Method. Journal of the Electrochemical Society, 157(11):

A1188-A1195.

Publisher's Version: http://dx.doi.org/10.1149/1.3486082

This Article is brought to you by the Chemical Engineering, Department of at Scholar Commons. It has been accepted for inclusion in Faculty Publications by an authorized administrator of Scholar Commons. For more information, please contact digres@mailbox.sc.edu. 


\title{
(4HS) \\ An Efficient Electrochemical-Thermal Model for a Lithium-Ion Cell by Using the Proper Orthogonal Decomposition Method
}

\author{
Long Cai* and Ralph E. White ${ }^{* *, z}$ \\ Department of Chemical Engineering, University of South Carolina, Columbia, South Carolina 29208, USA
}

The proper orthogonal decomposition method was applied to develop an efficient, reduced order electrochemical-thermal model for a lithium-ion cell. This model was validated for discharge simulations over a wide range of $\mathrm{C}$ rates and various cooling conditions of the cell. The reduced order model agrees well with the COMSOL model, a commercial finite element method solver, and requires $\sim 7$ times less computation time than the COMSOL model. The model predictions indicate that the discharge time or percent of capacity removed from the cell at an end of discharge voltage of $3.0 \mathrm{~V}$ depends on the rate of the discharge and heat transfer rate away from the cell. Also, the heat transfer rate determines whether the capacity removed is limited by mass transfer in the solid phase or mass transfer in the electrolyte.

(c) 2010 The Electrochemical Society. [DOI: 10.1149/1.3486082] All rights reserved.

Manuscript submitted May 10, 2010; revised manuscript received August 5, 2010. Published September 10, 2010.

Numerous simulation studies have been conducted to characterize the thermal behavior of lithium-ion batteries. A lumped electrochemical-coupled thermal model for the lithium aluminum and iron sulfide battery was developed by Pollard and Newman, ${ }^{1}$ where the temperature was assumed to be uniform throughout the cell sandwich and changed with time only, the heat generation rate consisted of the ohmic heat, reversible heat, heat exchange with the environment, and the heat of precipitation of electrolyte. This model was extended by involving the phase change and applying to batteries system ${ }^{2}$ and was also applied in the modeling of lithium/polymer battery in single cell, ${ }^{3}$ cell stack, ${ }^{4}$ and in the electric vehicle application with two- ${ }^{5}$ and three-dimensional ${ }^{6}$ simulation. In the two- ${ }^{5}$ and three-dimensional thermal models, ${ }^{6}$ the temperature was solved in two and three dimensions, respectively, whereas the electrochemical model was locally averaged. Starting from the first principlesbased electrochemical model ${ }^{7,8}$ for a lithium-ion battery, $\mathrm{Gu}$ and Wang ${ }^{9}$ developed the electrochemical-coupled distributed thermal model for the $\mathrm{LiCoO}_{2} /$ graphite cell. Their simulation results showed that the heat significantly affected the cell behavior during the discharge process. Similarly, Kumaresan et al. ${ }^{10}$ presented a thermal model for a lithium-ion cell and compared their model to the experimental data.

Because the first principles-based electrochemical-thermal model (also referred to as the pseudo-two-dimensional model, P2D) is a multiscale model, the computational time becomes a bottleneck for the system level and high dimensional simulation. To reduce the computation time, $\mathrm{Gu}$ and Wang ${ }^{9}$ used the diffusion length method and Kumaresan et al. ${ }^{10}$ used a two-term polynomial approximation method to simplify the diffusion equation of lithium ions in the solid phases of the active material in the electrodes. Subramanian et al. ${ }^{11}$ applied a reformulated model to reduce the number of equations. Smith and Wang ${ }^{12}$ applied a lumped thermal model in their simulation of a lithium-ion battery pack for hybrid electric vehicles. Smith et al. ${ }^{13}$ applied an equivalent circuit model and coupled it with a lumped thermal model to model a module with 16 cells in parallel. Cai and White ${ }^{14}$ used a proper orthogonal decomposition (POD) method to reduce the computation time.

This paper extends our previous work ${ }^{14}$ by adding an energy balance. The main purpose of the POD method is to reduce the computation time by decomposing "snapshots," which are obtained either from the experimental data or from the numerical simulation using a rigorous model, to obtain a set of orthonormal modes. These orthonormal modes are called proper orthogonal modes (POM) and capture the information of the snapshots in an optimal manner for a

\footnotetext{
* Electrochemical Society Student Member.

** Electrochemical Society Fellow.

z E-mail: white@cec.sc.edu
}

given number of eigenfunctions. The POD associated with the snapshot method ${ }^{15}$ has been widely used in the studies of turbulent flow, image processing, signal analysis, and oceanography to obtain a reduced order model (ROM) to save computational time. Our previous work ${ }^{14}$ showed that the POD associated with snapshot method gives a good approximation to the $\mathrm{P} 2 \mathrm{D}$ isothermal model not only in the overall cell voltage but also in the concentration and potential distribution along the cell and reduces the computation time by $\sim 7$ times. This paper is organized as follows: The thermal model for a lithium-ion cell is presented, followed by an explanation of the reduced order electrochemical-thermal lithium-ion cell model using a POD-associated snapshot method. Finally, this ROM is validated by comparing the prediction from the ROM to the rigorous model solved in COMSOL for example.

\section{Thermal Model}

Figure 1 presents the schematic of the cell sandwich that is modeled here. A one-dimensional energy balance for the single cell sandwich is given by

$$
\rho C_{\mathrm{p}} \frac{d T}{d t}=\frac{\partial}{\partial x}\left(\lambda \frac{\partial T}{\partial x}\right)+Q_{\mathrm{rxn}}+Q_{\mathrm{rev}}+Q_{\mathrm{ohm}}
$$

In Eq. 1, $T$ is the cell temperature, $\rho$ is the lumped density of the cell, $\lambda$ is the lumped thermal conductivity of the cell, $C_{\mathrm{p}}$ is the lumped specific heat, $Q_{\mathrm{rxn}}$ is the heat generation rate due to the electrochemical reaction, $Q_{\text {rev }}$ is the reversible heat, and $Q_{\mathrm{ohm}}$ is ohmic heat. In the one-dimensional geometry, $x$ is the spatial coordinate and the current direction passing in the cell during charge and is shown in Fig. 1. As shown in Fig. 1, $x$ starts from the interface of the current collector and the positive electrode and $L_{\mathrm{p}}$ is the thickness of positive electrode, $L_{\mathrm{s}}$ the thickness of the separator, and $L_{\mathrm{n}}$ the thickness of the negative electrode.

Assuming this single cell sandwich is exposed to the environment, then the boundary conditions can be determined by using Newton's cooling law

$$
\begin{gathered}
\text { at } x=0-\left.\left(\lambda \frac{\partial T}{\partial x}\right)\right|_{x=0}=h\left(T_{\text {air }}-T\right) \\
\text { at } x=L_{\mathrm{p}}+L_{\mathrm{s}}+L_{\mathrm{n}}-\left.\left(\lambda \frac{\partial T}{\partial x}\right)\right|_{x=L_{\mathrm{p}}+L_{\mathrm{s}}+L_{\mathrm{n}}}=h\left(T-T_{\text {air }}\right)
\end{gathered}
$$

where $h$ is the effective heat transfer coefficient and $T_{\text {air }}$ denotes the temperature of the environment, the heat sources in Eq. 1 can be written as follows

$$
Q_{\mathrm{rxn}}=F a J\left(\phi_{1}-\phi_{2}-U\right)
$$




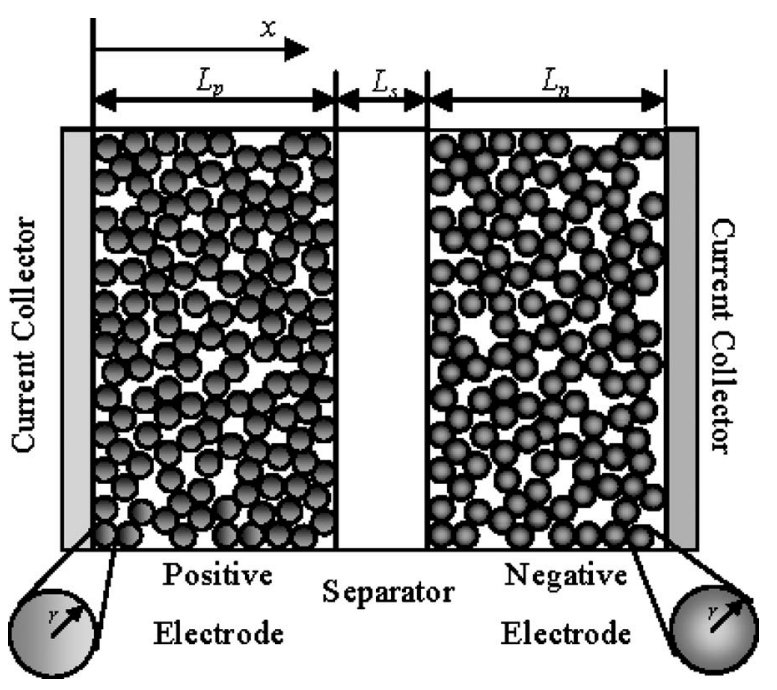

Figure 1. Schematic of a lithium-ion cell.

$$
\begin{gathered}
Q_{\text {rev }}=F a J T\left(\frac{\partial U}{\partial T}\right) \\
Q_{\text {ohm }}=\sigma_{\text {eff }}\left(\frac{\partial \phi_{1}}{\partial x}\right)^{2}+\kappa_{\text {eff }}\left(\frac{\partial \phi_{2}}{\partial x}\right)^{2}+\frac{2 \kappa_{\mathrm{eff}} R T}{F}\left(1-t_{+}^{0}\right) \frac{1}{c_{\mathrm{e}}} \frac{\partial c_{\mathrm{e}}}{\partial x} \frac{\partial \phi_{2}}{\partial x}
\end{gathered}
$$

In Eq. $2-4, F$ is Faraday's constant, $a$ is the specific area of the porous electrodes, $J$ is the pore wall flux, $\phi_{1}$ is the potential in the solid phase, $\phi_{2}$ is the potential in the binary electrolyte, and $c_{\mathrm{e}}$ is the concentration of the binary electrolyte. The governing equations for $\phi_{1}, \phi_{2}$, and $c_{\mathrm{e}}$ are presented in Ref. 7-9 and 14. The temperaturedependent open-circuit potential of the electrode i $\left(U_{\mathrm{i}}\right)$ can be approximated by Taylor's first-order expansion around a reference temperature

$$
U_{\mathrm{i}}=U_{\text {ref }, \mathrm{i}}+\left.\left(T-T_{\text {ref }}\right)\left(\frac{d U}{d T}\right)_{\mathrm{i}}\right|_{T_{\text {ref }}} \mathrm{i}=\mathrm{p}, \mathrm{n}
$$

where $U_{\text {ref,i }}$ is the open-circuit potential under the reference temperature $T_{\text {ref }}$ and is a function of state of charge of the electrodes, $\left.(d U / d T)_{\mathrm{i}}\right|_{T_{\text {ref }}}$ indicates the entropy of the intercalation reaction of $\mathrm{Li}$ ions in the $i$ electrode evaluated at the reference temperature. The pore wall flux of the active material particles in electrode $i$ is given by the Butler-Volmer equation as follows

$$
\begin{aligned}
J_{\mathrm{i}}= & k_{\mathrm{i}}\left(c_{\mathrm{s}, \mathrm{i}, \max }-c_{\mathrm{s}, \mathrm{i}, \text { surf }}\right)^{0.5} c_{\mathrm{s}, \mathrm{i}, \text { surf }}^{0.5} c_{\mathrm{e}, \mathrm{i}}^{0.5}\left[\exp \left(\frac{0.5 F}{R T} \eta_{\mathrm{i}}\right)\right. \\
& \left.-\exp \left(-\frac{0.5 F}{R T} \eta_{\mathrm{i}}\right)\right] \quad \mathrm{i}=\mathrm{p}, \mathrm{n}
\end{aligned}
$$

In Eq. $6, k_{\mathrm{i}}$ is the reaction rate constant of the electrochemical reaction that occurs on the interface of the active material particle and the electrolyte, $c_{\mathrm{s}, \mathrm{i} \text { max }}$ is the maximum concentration of $\mathrm{Li}$ ions in the active material of the electrode $\mathrm{i}, c_{\mathrm{s}, \mathrm{i} \text {,surf }}$ is the concentration of $\mathrm{Li}$ ions on the surface of the active material particles, and $R$ is the universal gas constant. The overpotential for electrode $\mathrm{i}$ is determined by

$$
\eta_{\mathrm{i}}=\phi_{1, \mathrm{i}}-\phi_{2, \mathrm{i}}-U_{\mathrm{i}}
$$

The temperature-dependent diffusion coefficient, $D_{\mathrm{e}, \mathrm{i}}$, and ionic conductivity, $\kappa_{\mathrm{i}}$, of the $\mathrm{LiPF}_{6}$ in propylene carbonate/ethylene carbonate/dimethyl carbonate in the cell component $\mathrm{i}$ are expressed by $^{16}$

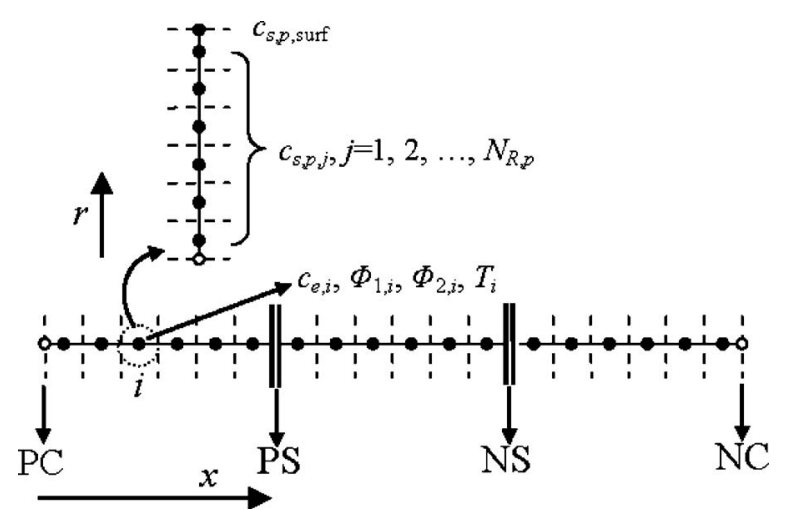

Figure 2. Schematic for the FVM for the multiscale cell thermal model (with interfaces indicated: PC, PS, NS, and NC).

$$
\begin{aligned}
& D_{\mathrm{e}, \mathrm{i}}=1 \times 10^{-4} \times 10^{-4.43-} \frac{54}{T-229-5.0 \times 10^{-3} c_{\mathrm{e}, \mathrm{i}}}-0.22 \times 10^{-3} c_{\mathrm{e}, \mathrm{i}} \\
\kappa_{\mathrm{i}}= & 10^{-4} \times c_{\mathrm{e}, \mathrm{i}}\left(-10.5+0.668 \times 10^{-3} c_{\mathrm{e}, \mathrm{i}}+0.494 \times 10^{-6} c_{\mathrm{e}, \mathrm{i}}^{2}\right. \\
& +0.074 T_{\mathrm{i}}-1.78 \times 10^{-5} c_{\mathrm{e}, \mathrm{i}} T_{\mathrm{i}}-8.86 \times 10^{-10} c_{\mathrm{e}, \mathrm{i}}^{2} T_{\mathrm{i}}-6.96 \\
& \left.\times 10^{-5} T_{\mathrm{i}}^{2}+2.80 \times 10^{-8} c_{\mathrm{e}, \mathrm{i}} T_{\mathrm{i}}^{2}\right)^{2}
\end{aligned}
$$

where $\mathrm{i}=\mathrm{p}, \mathrm{s}$, and $\mathrm{n}$ and the diffusion coefficient, ionic conductivity, electrolyte concentration, and temperature are in the units $\mathrm{m}^{2} / \mathrm{s}$, $\mathrm{S} / \mathrm{m}, \mathrm{mol} / \mathrm{m}^{3}$, and $\mathrm{K}$, respectively.

The temperature-dependent diffusion coefficient of Li ions in the solid phase, $D_{\mathrm{s}}$, and the reaction rate constant, $k$, are approximated by using an Arrhenius type expression

$$
\varphi=\varphi_{\text {ref }} \exp \left[\frac{E a^{\varphi}}{R}\left(\frac{1}{T_{\text {ref }}}-\frac{1}{T}\right)\right]
$$

where $\varphi$ denotes $D_{\mathrm{s}}$ and $k, E a^{\varphi}$ is the activation energy of $\varphi$, and $\varphi_{\text {ref }}$ is the value of $\varphi$ at the reference temperature $T_{\text {ref }}$.

The first principles-based electrochemical-thermal model mentioned above is solved numerically using the finite volume method (FVM) in FORTRAN. For comparison purpose, the same equations are also solved in COMSOL, a commercial software package that is designed for a multidiscipline simulation using the finite element method. Figure 2 shows the discretized multiscale geometry for a lithium-ion cell. In the macroscale ( $x$-direction), the finite volumes are assigned in the component-wise so that the interfaces (double vertical lines shown in Fig. 2) of the positive electrode/the separator and the separator/the negative electrode are also the surfaces of the corresponding finite volumes. Because the one-dimensional model is considered in this work, the finite volume is a line segment that is enclosed by two dashed vertical lines, which indicate the surfaces of the finite volume. The node point is located at the center of the finite volume and denoted by a solid point in Fig. 2. The equations are only solved on these node points besides the cell voltage and the concentration of the lithium ion on the particle surface, which are solved in the microscale. The cell voltage is the difference of the solid-phase potentials at the two ends of the cell, which are indicated by the two open circles in Fig. 2. The concentration of lithium ions on the surface of the particle is involved in Butler-Volmer equation (Eq. 6) and needs to be solved explicitly using a three-point backward difference method. In this work, the electrodes in the $x$-direction are divided into 100 finite volumes. The separator contains 70 volumes and the particles in the $r$-direction are partitioned to 50 finite volumes. In this manner, there are totally $(100+70$ $+100) \times(4+51)=14,850$ equations to be solved. To keep a banded coefficient matrix, the equations for the concentration and the potential in the solid phase are also included in the separator (though do not exist there) to improve the computation speed. We denote by PC, PS, NS, NC the interfaces between the current col- 
lector and the positive electrode, the positive electrode and the separator, the separator and the negative electrode, and the negative electrode and the current collector, respectively, as shown in Fig. 2. The physical model is discretized in the spatial coordinates only; thus, the resulting model consists of a set ordinary differential equations (with respect to time) and algebraic equations. We refer to this model as the rigorous model, which is denoted by FVM in this paper.

\section{POD-based Reduced Thermal Model}

The model reduction based on the POD is to find a nonhomogeneous state space form for the dependent variables. POD is a procedure to find a basis for a modal decomposition of an ensemble of signals. Mathematically, POD corresponds to finding the basis or set of eigenfunctions in Hilbert space $L^{2}$ that satisfies the following constrained optimization problem ${ }^{17}$

$$
\max _{\phi \in L^{2}}\left\langle|(u, \phi)|^{2}\right\rangle \quad \text { subject to }(\phi, \phi)=1
$$

where $u(x)$ is the ensemble of signals and $\phi(x)$ are the basis functions, where $\phi(x) \in L^{2}(\Omega)$ and $x \in \Omega .\langle\cdot\rangle$ denotes the time average, and $|\cdot|$ denotes the modulus. The inner product of $u$ and $\phi$ in the $L^{2}$ space is defined by $(u, \phi)=\int_{\Omega} u(x) \phi^{*}(x) d x$, where the superscript ${ }^{*}$ refers to the complex conjugate of $\phi(x)$. The constraint shown in Eq. 11 merely serves to normalize the functions $\phi(x)$ and thus, ensures the uniqueness of the solution. The problem defined in Eq. 11 is equivalent to maximizing the functional

$$
L(\phi, \lambda)=\left\langle|(u, \phi)|^{2}\right\rangle-\lambda[(\phi, \phi)-1]
$$

where $\lambda$ is Lagrange multiplier. Extrema of the function $L(\phi, \lambda)$ can be found by setting its Gateaux derivative to zero ${ }^{17}$

$$
\left.\frac{\partial}{\partial \delta} L(\phi+\delta \psi, \lambda)\right|_{\delta=0}=0
$$

where $\delta$ is a real number and $\psi$ is an arbitrary function in $L^{2}$ space. Solving Eq. 13 leads to

$$
\int_{\Omega}\left\langle u(x) u^{*}(y)\right\rangle \phi(y) d y=\lambda \phi(x)
$$

where $x$ and $y$ are the elements in $\Omega$. The problem defined in Eq. 14 is an infinite dimensional eigenvalue problem. The kernel of the eigenvalue problem is

$$
K(x, y)=\left\langle u(x) u^{*}(y)\right\rangle \quad \text { where } x, y \in \Omega
$$

which is positive definite and bounded. $K(x, y)$ is also known as the two-point correlation tensor. Equation 14 can be rewritten in more compact way as

$$
K \phi=\lambda \phi
$$

According to the Hilbert-Schmidt theorem, ${ }^{18}$ there exists a diagonal decomposition of kernel $K(x, y)$, which is given by

$$
K(x, y)=\sum_{i=1}^{\infty} \lambda_{i} \phi_{i}(x) \phi_{i}^{*}(y)
$$

where $\phi_{i}(x)$ and $\lambda_{i}$ are the eigenfunction/eigenvalue pair of the kernel $K$ and $\lambda_{i} \geq 0$, respectively. This set of eigenfunctions, $\left\{\phi_{i}(x)\right\}_{i=1}^{\infty}$, forms a complete orthonormal basis in $L^{2}$ space. Every member of the ensemble may be reproduced by a modal decomposition using these eigenfunctions

$$
u(x)=\sum_{i=1}^{\infty} a_{i} \phi_{i}(x)
$$

The magnitude of the kernel $K$ in some sense denotes the total energy of the dynamic system. ${ }^{17}$ The square norm of $K$ can be determined by

$$
\|K(x, y)\|^{2}=\int_{\Omega} \int_{\Omega}|K(x, y)|^{2} d x d y=\sum_{i=1}^{\infty} \lambda_{i}^{2}
$$

Equation 19 indicates that the total energy of the ensemble is the sum of the square of the eigenvalues of $K$. Every eigenvalue indicates the importance of the corresponding eigenfunction to the representation of the ensemble. Consequently, we can sort the eigenvalue/eigenfunction pair by $\lambda_{i} \geq \lambda_{i+1}$ and choose the first $N$ eigenfunctions to form a subspace $\left\{\phi_{i}(x)\right\}_{i=1}^{N}$. The projection of the signal $u(x, t)$ of the ensemble onto this subspace is given by

$$
\hat{u}(x, t)=\sum_{i=1}^{N} a_{i}(t) \phi_{i}(x)
$$

The optimality of the POD that is verified in Ref. 17 and 18 states that

1. The POD coefficients $a_{i}(t)$ are uncorrelated so that $\left\langle a_{i}(t) a_{\mathrm{j}}^{*}(t)\right\rangle=\delta_{i \mathrm{j}} \lambda_{i}$.

2. The infinite set of functions $\left\{\psi_{i}(x)\right\}_{i=1}^{\infty}$ can be an arbitrary orthonormal set such that $u(x, t)=\sum_{i=1}^{\infty} b_{i}(t) \psi_{i}(x)$. For every $N$ we have $\sum_{i=1}^{N}\left\langle a_{i}(t) a_{i}^{*}(t)\right\rangle=\sum_{i=1}^{N} \lambda_{i} \geq \sum_{i=1}^{N}\left\langle b_{i}(t) b_{i}^{*}(t)\right\rangle$.

The second statement is the basis for the claim that the POD is optimal for modeling or reconstructing a signal $u(x, t)$. Among all linear decompositions, Eq. 20 is the most compact in the sense that for a given number of modes $(N)$, the projection on the subspace $\left\{\phi_{i}(x)\right\}_{i=1}^{N}$ contains the most "kinetic energy" on the average. For convenience, we name the POD coefficients $a_{\mathrm{i}}(t)$ the reduced variables associated with $u(x, t)$ and the elements in the subspace $\phi_{\mathrm{i}}(x)$ the POMs.

For the cases where the analytical solutions for $\phi(x)$ cannot by solved from Eq. 16, a numerical scheme is required. A data ensemble $\mathbf{Y}$ can be prepared from a set of vector valued signals denoted by $y_{\mathrm{j}}=u(\underline{x}, j \tau) \in \mathbb{R}^{m}$ from the solution of $u$ evaluated on the $m$ discrete spatial node points $\underline{x}$ at the $j$ th time step $(j \tau)$, and is an $m \times n$ matrix, $Y=\left[\underline{y}_{1}, \underline{y}_{2}, \ldots, \underline{y}_{n}\right]$, where $n$ is the total number of time steps. The discrete form of the kernel defined in Eq. 15 is given by ${ }^{19}$

$$
\bar{K}=\frac{1}{n} Y Y^{\mathrm{T}}
$$

where $Y^{\mathrm{T}}$ is the transpose of $\mathbf{Y}$. A singular value decomposition (SVD) of the discrete kernel $\bar{K}$ yields

$$
\operatorname{SVD}(\bar{K})=\Phi \Sigma \Phi^{\mathrm{T}} \text { or } Y Y^{\mathrm{T}} \Phi=\Phi \Lambda
$$

where $\Lambda=\Sigma / n$. In Eq. 22, $\Phi$ is an $m \times m$ matrix, and $\Sigma$ is an $m$ $\times m$ diagonal matrix. The nonzero diagonal elements in $\Sigma$ are in the order of $\sigma_{1} \geq \sigma_{2}, \ldots, \geq \sigma_{d}>0$, where $d=\min (m, n)$ (note that $\left.\lambda_{\mathrm{i}}=\sigma_{\mathrm{i}} / n\right)$. The column vectors in $\Phi$ form an orthonormal basis and are the POMs in $\mathbb{R}^{m}$ space.

The diagonal decomposition defined in Eq. 22 is an $m$-order eigenvalue problem. If the number of spatial node points is large, it is difficult to determine the POMs by solving Eq. 22. If $m>n$ (for instance in the P2D diffusion equation of the lithium-ion battery model, $m=5000$ and $n$ is about tens to hundreds), it is more convenient to convert the $m$-order eigenproblem to $n$-order eigenproblem if the number of time steps $n$ is much less than $m$. The method of snapshots ${ }^{15}$ provides an efficient way to conduct this conversion. The solution for the signal at each time step is arranged as a vector and named as a snapshot. The ensemble $\mathbf{Y}$ is a collection of these snapshots. The main idea of the method of snapshots is that the POMs are the linear combination of the snapshots

$$
\Phi=Y A
$$

where the ensemble $\mathbf{Y}$ is an $m \times n$ matrix, $m$ is the number of node 
points, $n$ is the number of snapshots, and the matrix $\mathbf{A}$ remains to be determined. Substitution of Eq. 23 into Eq. 22 yields an $n$ th-order eigenvalue problem

$$
Y^{\mathrm{T}} Y A=A \Lambda
$$

where $\mathbf{A}$ is the eigenvectors of matrix $Y^{\mathrm{T}} Y$. Finally, the POMs are determined by normalizing the column vector, $\phi_{j}$, and the $j$ th column of $\Phi$ by its own norm, that is

$$
\phi_{j}=\frac{\phi_{j}}{\left\|\phi_{j}\right\|} \quad j=1, \ldots, n
$$

The FVM (the rigorous model) presented in the previous section was solved at different current rates that range from $0.1 \mathrm{C}$ to $20 \mathrm{C}$. The simulation results of the dependent variables were recorded after every specified time interval so that the time points are $\sim 60$ for all the current rates. The POMs are determined such that every dependent variable corresponds to its own POMs. The snapshots for each variable, the simulation results obtained by solving the rigorous model, are molded into a two-dimensional matrix. For the variable solved in $x$-direction, such as $c_{\mathrm{e}}$ (the concentration of the electrolyte), $\phi_{1, i}$ (the solid-phase potential, i $=p, n$ ), $\phi_{2}$ (the potential in the electrolyte), $c_{\mathrm{s}, \mathrm{i} \text {,surf }}$ (the concentration of lithium on the surface of the particle, $\mathrm{i}=\mathrm{p}, \mathrm{n}$ ), and $T$ (the cell temperature), every row in its snapshot matrix is the spatial distribution at one-time step and every column in the snapshot matrix is the time changing at one node point in $x$-scale. The variables, such as $c_{\mathrm{s}, \mathrm{p}}$ (the concentration of lithium in the positive electrode) and $c_{\mathrm{s}, \mathrm{n}}$ (the concentration of lithium in the negative electrode), are a function of $r, x$, and time, so it is a P2D variable because the diffusion between particles was ignored. We calculate the POMs for $c_{\mathrm{s}, \mathrm{p}}$ and $c_{\mathrm{s}, \mathrm{n}}$ separately. At onetime step, the simulation solutions of the rigorous model for $c_{\mathrm{s}, \mathrm{p}}$ and $c_{\mathrm{s}, \mathrm{n}}$ are distributed in both $r$ - and $x$-scales. This one-time snapshot is in a two-dimensional data structure where the columns are generated in the $r$-direction and the rows are generated along the $x$-direction. This two-dimensional structure is reassembled to one column vector by column-wise. The snapshot matrix for $c_{\mathrm{s}, \mathrm{p}}$ and $c_{\mathrm{s}, \mathrm{n}}$ consists of these column vectors that correspond to the multiple-time steps. Therefore, we have nine snapshot matrices for the nine variables: $c_{\mathrm{s}, \mathrm{p} \text { surf }}, c_{\mathrm{s}, \mathrm{n}, \mathrm{surf}}, c_{\mathrm{e}}, \phi_{1, \mathrm{p}}, \phi_{1, \mathrm{n}}, \phi_{2}, T, c_{\mathrm{s}, \mathrm{p}}$, and $c_{\mathrm{s}, \mathrm{n}}$. Directly applying SVD to the first seven snapshot matrices, which correspond to the first seven variables listed previously, yields the seven sets of POMs. Because $c_{\mathrm{s}, \mathrm{p}}$ and $c_{\mathrm{s}, \mathrm{n}}$ are the two-dimensional variables, the snapshot method was applied for these two variables to obtain the POMs for them.

Applying the second optimality of the POMs, we took the first few columns from the full set of POMs for each variable to form a subspace and constructed the ROM on this subspace. Let us take the diffusion equation in the electrolyte as an example to illustrate the procedures to formulate its reduced order form. In the rigorous model, the concentration of the electrolyte is presented by a vector $C_{\mathrm{e}}(t)$ with 270 items corresponding to the 270 finite volumes in $x$-direction illustrated in Fig. 2, which are functions of time. By Galerkin's approximation, $C_{\mathrm{e}}(t)$ can be approximated by the linear combination of the first few (for example $N_{\text {ce }}$ ) columns of its POMs

$$
\underline{C_{\mathrm{e}}}(t) \doteq \sum_{i=1}^{N_{\mathrm{ce}}} a_{\mathrm{ce}, i}(t) \underline{\phi_{\mathrm{ce}, i}}=\underline{\underline{\Phi_{\mathrm{ce}}}} \underline{a_{\mathrm{ce}}}(t)
$$

where $\Phi_{\text {ce }}$ is a $270 \times N_{\text {ce }}$ matrix and is composed of the column

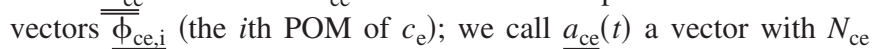
items $a_{\mathrm{ce}, i}(t)$, where $i=1, \ldots, N_{\mathrm{ce}}$, the reduced variable associated with $C_{\mathrm{e}}(t)$. Generally, $N_{\mathrm{ce}}$ is much smaller than the 270 dimension of $\underline{C_{\mathrm{e}}} \overline{(t)}$. In the rigorous model obtained using FVM, $\underline{C_{\mathrm{e}}}(t)$ is governed by

$$
\frac{d \underline{C_{\mathrm{e}}}(t)}{d t}=\underline{\underline{A_{\mathrm{ce}}}} \underline{\underline{C_{\mathrm{e}}}}(t)+\underline{B_{\mathrm{ce}}} \text { and initially } \underline{C_{\mathrm{e}}}(0)=\underline{c_{0}}
$$

where $A_{\mathrm{ce}}$ is the coefficient matrix and can be a function of $x$ and other dependent variables, $B_{\mathrm{ce}}$ is the source vector, and $c_{0}$ is the vector consists of the initial values on the node points. Substituting Eq. 26 into Eq. 27 yields

$$
\underline{\underline{\Phi_{\mathrm{ce}}}} \frac{d a_{\mathrm{ce}}(t)}{d t}=\underline{\underline{A_{\mathrm{ce}}}} \underline{\underline{\Phi_{\mathrm{ce}}}} \underline{\underline{a_{\mathrm{ce}}}}(t)+\underline{B_{\mathrm{ce}}}
$$

Projecting Eq. 28 to the subspace $\Phi_{\text {ce }}$ and applying the property of the orthonormality of the subspace, we have

$$
\frac{d \underline{a_{\mathrm{ce}}(t)}}{d t}=\underline{\underline{\Phi_{\mathrm{ce}}}} \underline{\underline{A_{\text {ce }}}} \underline{\underline{\Phi_{\text {ce }}}} \underline{\underline{a_{\mathrm{ce}}}}(t)+\underline{\underline{\Phi_{\mathrm{ce}}^{\mathrm{T}}}} \underline{\underline{B_{\mathrm{ce}}}}
$$

The superscript "T" in Eq. 29 denotes the matrix transpose. Equation 29 is the reduced order form of Eq. 27. The initial condition for the reduced variable is given by

$$
\underline{a}_{\mathrm{ce}}(0)=\underline{\underline{\Phi_{\mathrm{ce}}}} \underline{c}^{\mathrm{T}}
$$

For the variables that explicitly depend on time (time derivative of the variable appears in its governing equation), such as $c_{\mathrm{e}}, c_{\mathrm{s}, \mathrm{p}}$, $c_{\mathrm{s}, \mathrm{n}}$, and $T$, their ROMs are obtained following the above procedures. For the variables $c_{\mathrm{s}, \mathrm{p}, \text { surf }}, c_{\mathrm{s}, \mathrm{n}, \mathrm{surf}}, \phi_{1, \mathrm{p}}, \phi_{1, \mathrm{n}}$, and $\phi_{2}$, their time derivatives are not involved in their governing equations. The ROMs associated with those variables are illustrated by taking $\phi_{1, \mathrm{p}}$ as an example. The rigorous model for $\phi_{1, \mathrm{p}}$ is given by

$$
\underline{\underline{A_{\mathrm{f} 1 \mathrm{p}}}} F_{1, \mathrm{p}}(t)=\underline{B_{\mathrm{f} 1 \mathrm{p}}}
$$

where $F_{1, \mathrm{p}}(t)$ is the vector consists of the solid-phase potentials at the node points in the positive electrode and implicitly depends on time by coupling with other dependent variables, $A_{\mathrm{flp}}$ is the coefficient matrix, and $\underline{B}_{\mathrm{flp}}$ is the source vector. The subspace for $\phi_{1, \mathrm{p}}$ is formed by choosing the first few $\left(N_{\mathrm{f} 1 \mathrm{p}} \ll 100\right)$ columns from its full set of POMs and denoted by $\Phi_{\mathrm{fl} p}$, which is a $100 \times N_{\mathrm{f} 1 \mathrm{p}}$ matrix. The Galerkin's approximation of $F_{1, \mathrm{p}}(t)$ is given by

$$
\underline{F_{1, \mathrm{p}}}(t) \doteq \underline{\underline{\Phi_{\mathrm{flp}}}} \underline{a_{\mathrm{flp}}}(t)
$$

where $a_{\mathrm{f} 1 \mathrm{p}}(t)$ is the vector of the reduced variable associated to $F_{1, \mathrm{p}}(t)$. Substituting Eq. 32 into Eq. 31, we have

$$
\underline{\underline{A_{\mathrm{f} 1 \mathrm{p}}}} \underline{\underline{\Phi_{\mathrm{f} 1 \mathrm{p}}}} \underline{a_{\mathrm{f} 1 \mathrm{p}}}(t)=\underline{B_{\mathrm{f} 1 \mathrm{p}}}
$$

Projecting Eq. 33 to $\underline{\underline{\Phi_{\text {f1p }}}}$ yields

$$
\underline{\underline{\Phi_{\mathrm{fl} 1 \mathrm{p}}}} \underline{\underline{{ }^{\mathrm{T}}}} \underline{\underline{A_{\mathrm{f} 1 \mathrm{p}}}} \underline{\underline{\Phi_{\mathrm{fl} 1 \mathrm{p}}}} \underline{a_{\mathrm{flp}}}(t)=\underline{\underline{\Phi_{\mathrm{flp}}}} \underline{{ }^{\mathrm{T}}} \underline{B_{\mathrm{flp}}}
$$

Equation 34 is the ROM for $F_{1, \mathrm{p}}(t)$. The same procedures can be followed to calculate the ROMs for the implicitly time-dependent variables.

In the thermal model presented in the previous section, the cell temperature is coupled to concentrations of lithium and the potentials in the solid phase and the electrolyte through the temperaturedependent transport parameters: diffusion coefficients of lithium, reaction rate constants, and ionic conductivity. The transport patterns for these variables are much more complicated compared to those in the isothermal case in our previous work. ${ }^{14}$ As a result, more POMs are needed in Galerkin's approximation to represent the dependent variables in the rigorous model. In this work, the dimensions of the reduced variable vectors are assigned as $9,11,8,9,10,4,9,9$, and 1 for $a_{\mathrm{cs}, \mathrm{p}}, a_{\mathrm{cs}, \mathrm{n}}, a_{\mathrm{cs}, \mathrm{p}, \mathrm{surf}}, a_{\mathrm{cs}, \mathrm{n}, \mathrm{sur}}, a_{\mathrm{ce}}, a_{\mathrm{f} 1 \mathrm{p}}, a_{\mathrm{f} 1 \mathrm{n}}, a_{\mathrm{f} 2}$, and $a_{\mathrm{T}}$, respectively. Totally, there are $\frac{\mathrm{cs,n, \textrm {sur }}}{70}$ equations involved in the ROM with thermal effect; in contrast, the isothermal ROM in our previous work $^{14}$ consists of 50 equations. Because the Biot number [Bi $\left.=h /\left(\lambda_{\mathrm{p}} / L_{\mathrm{p}}+\lambda_{\mathrm{s}} / L_{\mathrm{s}}+\lambda_{\mathrm{n}} / L_{\mathrm{n}}\right)\right]$ is very small, $B i=3.4 \times 10^{-5}$ when $h=2.0 \mathrm{~W} / \mathrm{m}^{2} / \mathrm{K}$, the temperature is almost uniform across the cell sandwich that one equation assigned for $\underline{a_{\mathrm{T}}}$ is sufficient enough. 


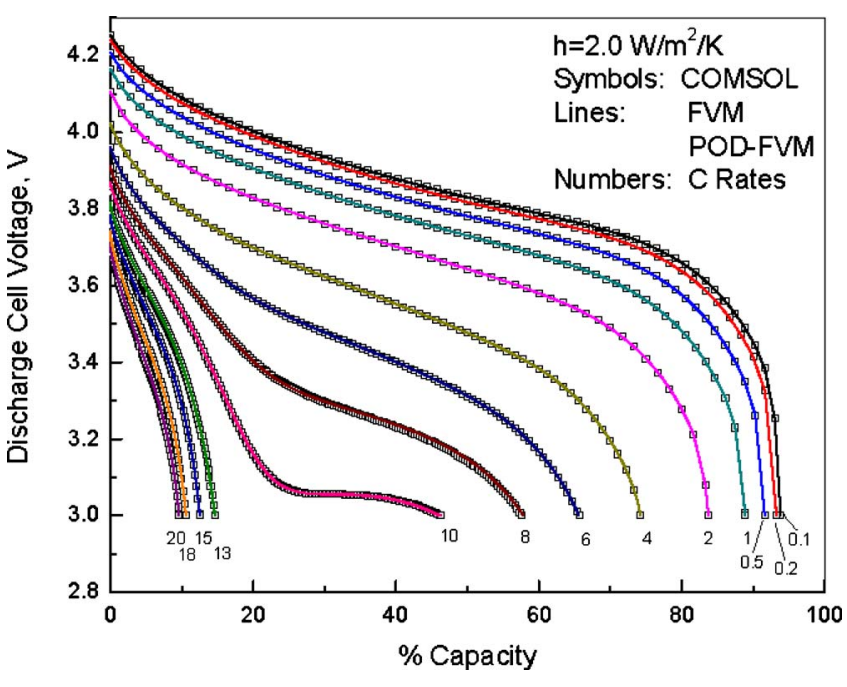

Figure 3. (Color online) Comparisons of the discharge cell voltage between the ROM, the rigorous model solved in FORTRAN program with FVM, and the physics model solved in COMSOL with FEM under various $\mathrm{C}$ rates changing from $0.1 \mathrm{C}$ to $20 \mathrm{C}$ with the effective heat transfer coefficient $(h)$ fixed at $2.0 \mathrm{~W} / \mathrm{m}^{2} / \mathrm{K}$.

\section{Results}

The ROM presented in the previous section is a set of differential algebraic equations (DAEs). An open-source DAE solver with zero crossing, DASRT written in FORTRAN, was used to solve our ROM using POD based on FVM (denoted by POD-FVM) and the rigorous model (denoted by FVM). The results of ROM were compared to those of the rigorous model with FVM and those of the COMSOL model with finite element method (FEM, denoted by COMSOL). The $\mathrm{C}$ rate current of the cell in this work is $1.656 \mathrm{~A}$. The cell parameters can be found in Ref. 10. For the same $\mathrm{C}$ rate discharge process, the same convergence criteria (both the relative and the absolute tolerances are $1.0 \times 10^{-6}$ in this work) and the same numbers of the time outputs, the ROM requires $10-12 \mathrm{~s}$ run on a personal computer with Pentium $43.6 \mathrm{GHz}$ central processing unit, 3.6 GHz, 2.0 G random access memory, and Window XP operational system. Run on the same computer with the same condition, the rigorous model solved in FORTRAN needs $\sim 40 \mathrm{~s}$ and the COMSOL model needs $\sim 75 \mathrm{~s}$.

Figure 3 shows the predicted discharged cell voltages for $\mathrm{C}$ rates from $0.1 \mathrm{C}$ to $20 \mathrm{C}$ between the POD-FVM, the FVM, and the COMSOL model in which the effective heat transfer coefficient of the air surrounding the cell is $2.0 \mathrm{~W} / \mathrm{m}^{2} / \mathrm{K}$. In the simulation, the cell was discharged starting from the fully charged state until the voltage reached the cutoff voltage, $3.0 \mathrm{~V}$. Figure 4 shows the cell temperature at $x=0$ (shown in Fig. 2) for the three models under the same conditions. As the $\mathrm{C}$ rate increases, the cell discharged at the higher $\mathrm{C}$ rate achieves a tendency with the higher surface cell temperature. Due to the mass-transfer limiting, the cell cannot obtain the same capacity when discharged up to the same cutoff voltage at the different $\mathrm{C}$ rates. The higher rate discharge results in a shorter discharge time but possess a higher heat generation rate. As a result of the competition between these two points, at the end of discharge, the cell discharged at $10 \mathrm{C}$ obtained the highest cell surface temperature when $h$ is equal to $2 \mathrm{~W} / \mathrm{m}^{2} / \mathrm{K}$. The discharge rate under which the cell obtains the highest surface temperature at the end of discharge, changes as the changing of the effective heat transfer coefficient. For each $\mathrm{C}$ rate discharge process, the simulation results of these three models are overlapped with each other in Fig. 3 and 4. Figure 5 presents the errors in the discharge cell voltage between the rigorous model and the COMSOL model and between the ROM and the COMSOL model for the $0.1 \mathrm{C}, 1 \mathrm{C}$, and $10 \mathrm{C}$ discharge processes at $h=2.0 \mathrm{~W} / \mathrm{m}^{2} / \mathrm{K}$. The error for the ROM (denoted by POD-FVM

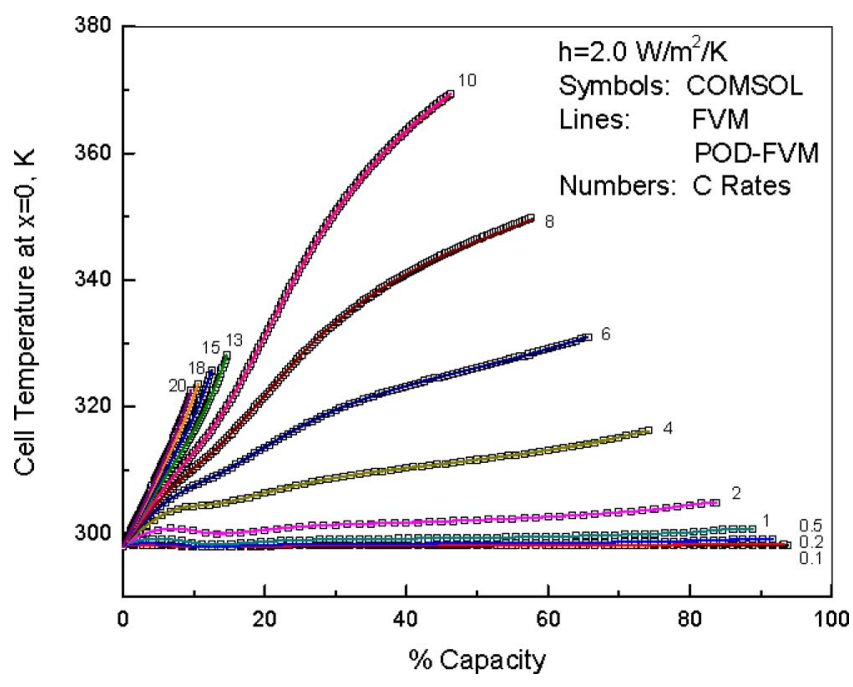

Figure 4. (Color online) Comparisons of the cell temperature at $x=0$ between the ROM, the rigorous model solved in FORTRAN program with FVM, and the physics model solved in COMSOL with FEM under various C rates changing from $0.1 \mathrm{C}$ to $20 \mathrm{C}$ with the effective heat transfer coefficient (h) fixed at $2.0 \mathrm{~W} / \mathrm{m}^{2} / \mathrm{K}$.

in the figure) compared to the COMSOL model, which is represented by the open symbols, is slightly higher than that of the rigorous model (denoted by FVM) indicated by the solid symbols, but is still less than $1 \mathrm{mV}$ for the $0.1 \mathrm{C}$ and $1 \mathrm{C}$ discharge cases and is no greater than $3 \mathrm{mV}$ for the $10 \mathrm{C}$ discharge process.

The cell performance also depends on the cooling conditions surrounding the cell. Figure 6 shows that the discharge cell voltage, obtained from the rigorous model and its ROM, changes with the different values for $h$ at the selected $\mathrm{C}$ rates: 1C, 10C, and 15C. Figure 6 consists of three groups of curves for the three selected $\mathrm{C}$ rates: $1 \mathrm{C}$ for the top right group, $15 \mathrm{C}$ for the bottom left group, and $10 \mathrm{C}$ for the middle group. In each group, there are four curves that correspond to the discharge voltages as a function of the discharge capacity at the four different cooling conditions where the effective heat transfer coefficient is equal to $0,1,2$, and $10 \mathrm{~W} / \mathrm{m}^{2} / \mathrm{K}$, respectively. In Fig. 6, the lines represent the results of the rigorous model and the symbols are the results of the ROM. The two models agree

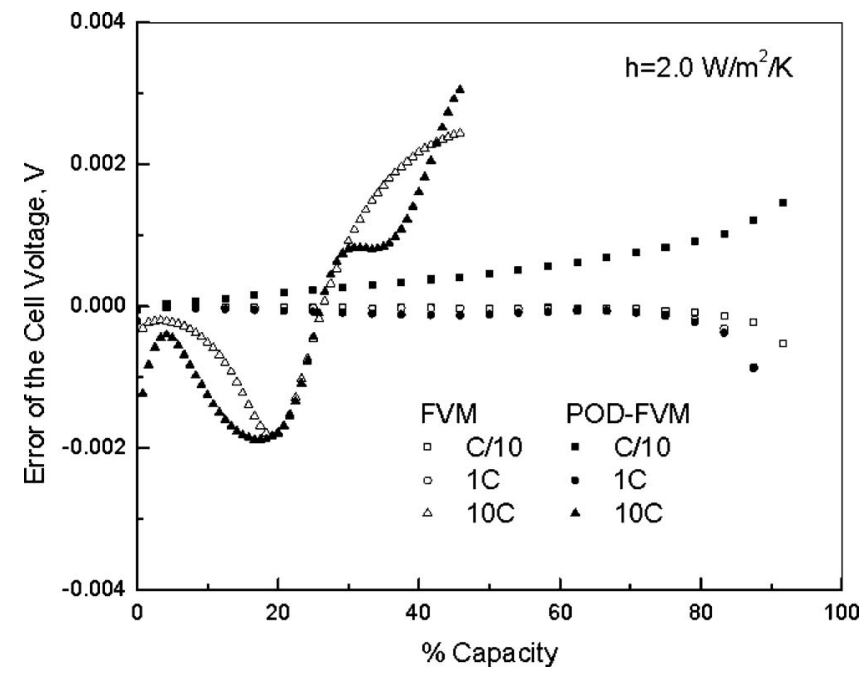

Figure 5. Errors in the discharge cell voltage between the ROM (denoted by POD-FVM) and the COMSOL model marked by the open symbols and between the rigorous model (denoted by FVM) and the COMSOL model marked by the solid symbols under the $\mathrm{C}$ rates: $0.1 \mathrm{C}, 1 \mathrm{C}$, and $10 \mathrm{C}$. 


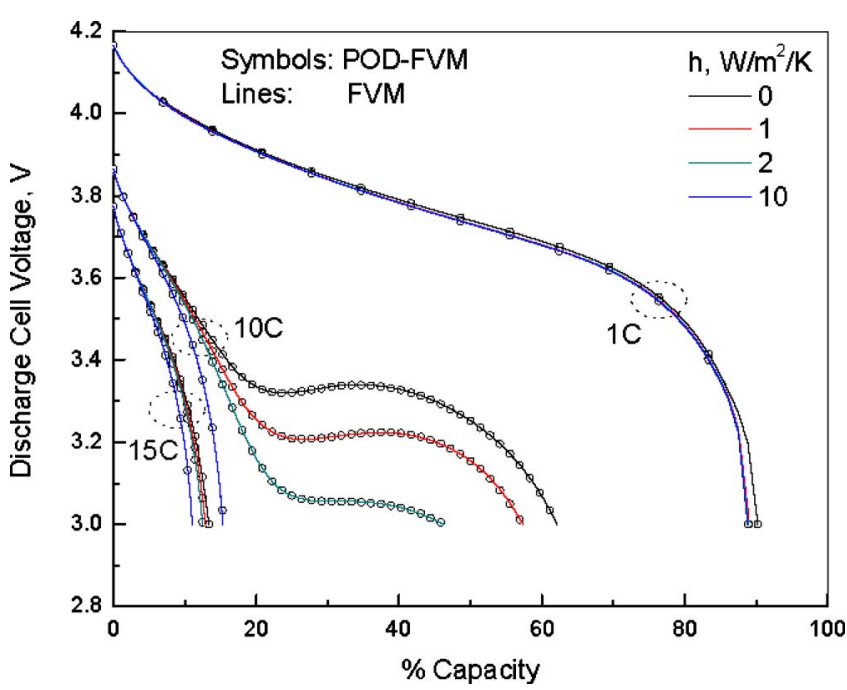

Figure 6. (Color online) Effect of the cooling condition on the voltage of the cell discharged at $1 \mathrm{C}, 10 \mathrm{C}$, and $15 \mathrm{C}$. Under each $\mathrm{C}$ rate, the effective heat transfer coefficient is chosen as $0,1,2$, and $10 \mathrm{~W} / \mathrm{m}^{2} / \mathrm{K}$. The symbols denote the solutions of the ROM, and the lines represent the solutions of the rigorous model.

well for all the cases shown in the figure. For all the three different $\mathrm{C}$ rates, the cell discharged under the adiabatic condition obtains the most discharge capacity and less and less as the value of $h$ increases. Figure 6 also shows that the effect of the cooling condition on the discharge cell voltage is not significant for the $1 \mathrm{C}$ discharge case and has moderate effect on the $15 \mathrm{C}$ discharge cases, but is very important for the $10 \mathrm{C}$ discharge process. Among the four curves in the $10 \mathrm{C}$ group, the discharge curve for $h$ equals to $10 \mathrm{~W} / \mathrm{m}^{2} / \mathrm{K}$ is way off the group, whereas the four curves in the other two groups follow the similar pattern. This can be explained by the thermal effect on the discharge behavior of the cell. The cell surface temperatures corresponding to the cases shown in Fig. 6 were presented in Fig. 7. The ROM yields good agreements of the cell surface temperature with the rigorous model for the cases shown in Fig. 7. In the $10 \mathrm{C}$ group, the cell surface temperature at the end of discharge is much smaller for the case where $h$ is equal to $10 \mathrm{~W} / \mathrm{m}^{2} / \mathrm{K}$

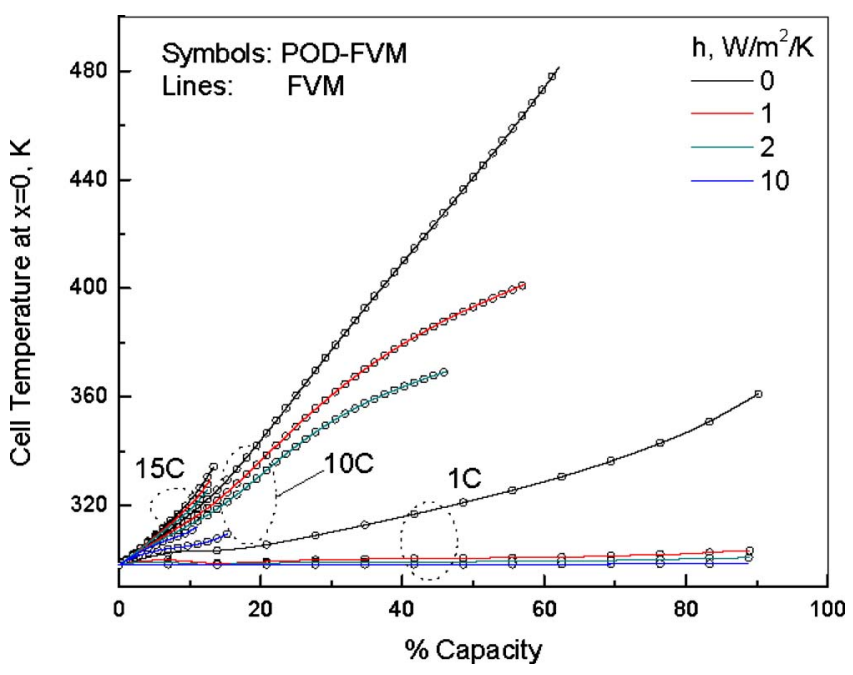

Figure 7. (Color online) Cell temperature at $x=0$ during the discharge process at $1 \mathrm{C}, 10 \mathrm{C}$, and $15 \mathrm{C}$. Under each $\mathrm{C}$ rate, the effective heat transfer coefficient is chosen as $0,1,2$, and $10 \mathrm{~W} / \mathrm{m}^{2} / \mathrm{K}$. The symbols denote the solutions of the ROM, and the lines represent the solutions of the rigorous model. than that in the case with $h=0,1$, and $2 \mathrm{~W} / \mathrm{m}^{2} / \mathrm{K}$. This is because the high temperature augmentation during the cell discharging with small effective heat transfer coefficient significantly improves the mass transfer in the cell, which results in more discharge capacity obtained for the cell discharged with this small $h$ value.

To scrutinize the thermal effect on the mass transfer in the electrolyte and the solid phase, the concentrations in the electrolyte and the surface concentration of the particles at the four interfaces marked in Fig. 2 are plotted in Fig. 8 and 9, respectively. In Fig. 8 and 9 , parts $\mathrm{a}, \mathrm{b}$, and $\mathrm{c}$ denote the cases for $1 \mathrm{C}, 10 \mathrm{C}$, and $15 \mathrm{C}$ discharge processes, respectively. In every graphs in Fig. 8 and 9, there are four groups of curves, each of which corresponds to one out of the four interfaces marked on the graphs. Each graph consists of four curves with different values for $h: 0,1,2$, and $10 \mathrm{~W} / \mathrm{m}^{2} / \mathrm{K}$. The symbols are the results of the ROM, and the lines are the results of the rigorous model. The graphs included in Fig. 8 and 9 indicate that the ROM follows the rigorous model very well. During the discharge process, lithium ions are extracted from the solid particles in the negative electrode, enter the electrolyte, move from the negative to the positive electrodes by passing through the separator, and are eventually inserted into the solid particles in the positive electrode. There are two possible controlling variables to end the discharge process (result in the cell voltage reaching the cutoff voltage): the concentration in the electrolyte at the interface PC and the surface concentration of the particle at the interface NS. Comparing the graphs in Fig. 8 and 9, at the end of discharge, the surface concentration of the particle at the interface between the separator and the negative electrode is close to zero, which indicates that for this low $\mathrm{C}$ rate discharge process, the mass diffusion in the solid phase is the limiting step. Inversely, for the high $\mathrm{C}$ rate discharge process (i.e., $15 \mathrm{C}$ or more), the concentration of the electrolyte at the interface between the current collector and the positive electrode is near zero at the end of discharge. Thus, for the high $\mathrm{C}$ rate discharge process, the mass transfer in the electrolyte is the limiting step. As mentioned in the previous paragraph, for 10C discharge, high cell temperature in the case with the low heat transfer coefficient of the surrounding cooling air improves the mass transfer in the electrolyte. This observation can be verified in Fig. 8b. As shown in Fig. 8b, when the discharge capacity is greater than $15 \%$ of the maximum capacity, the concentrations of the electrolyte at the interfaces PC, PS, and NS start to increase due to the cell temperature increasing.

The analysis presented in the previous paragraphs shows that the POD-based reduce order electrochemical-thermal model agrees well with the rigorous model. The accuracy of the POD-based ROM depends on the selection of the snapshots and the number of POMs chosen in the formulation of the ROM. To make the ROM valid for various conditions, we have to sample the simulation results obtained by running the rigorous model under different conditions to form the snapshots. The optimization of the selection of the snapshots is still an open question.

\section{Conclusion}

An effective reduced order electrochemical-thermal model for a lithium-ion cell was developed by using the POD based on the FVM. The ROM is 4 times faster than the rigorous model, which is solved in FORTRAN with FVM, and 7 times faster than the physical model solved in COMSOL with FEM.

The ROM was validated by comparing the rigorous and the COMSOL models in the simulation of discharge processes with a wide range of the $\mathrm{C}$ rates and different cooling conditions, which is represented by the varying of the value of the heat transfer coefficient of the surrounding cooling air. The comparisons of the discharge cell voltage, cell's surface temperature, surface concentration of the solid particles, and concentration of the electrolyte between the models revealed that the ROM based on the POD-FVM agrees well with the rigorous model.

The simulation results of the thermal model indicate that the low $\mathrm{C}$ rate discharge process is limited by the mass transfer in the solid 

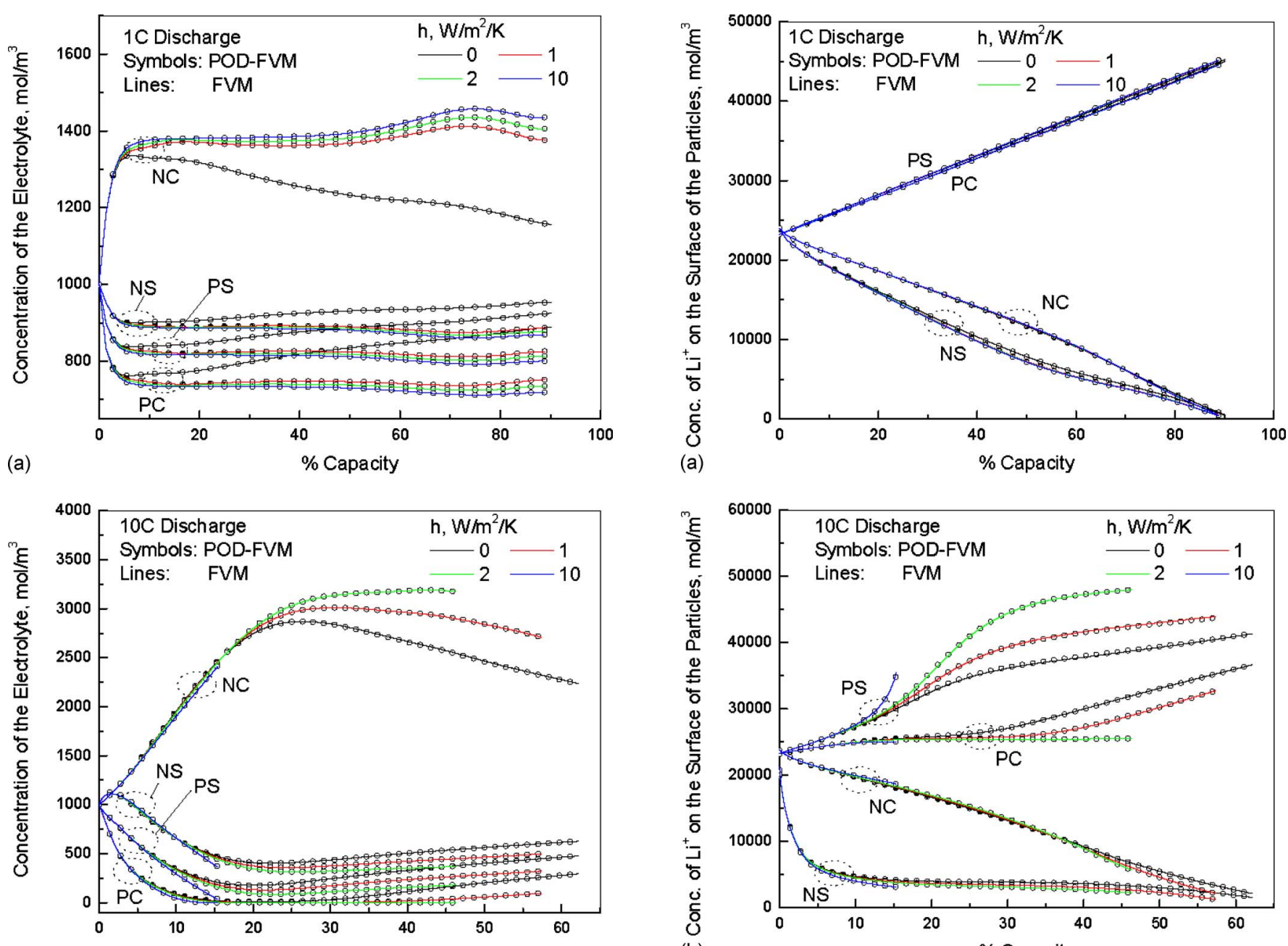

(b)

$\%$ Capacity
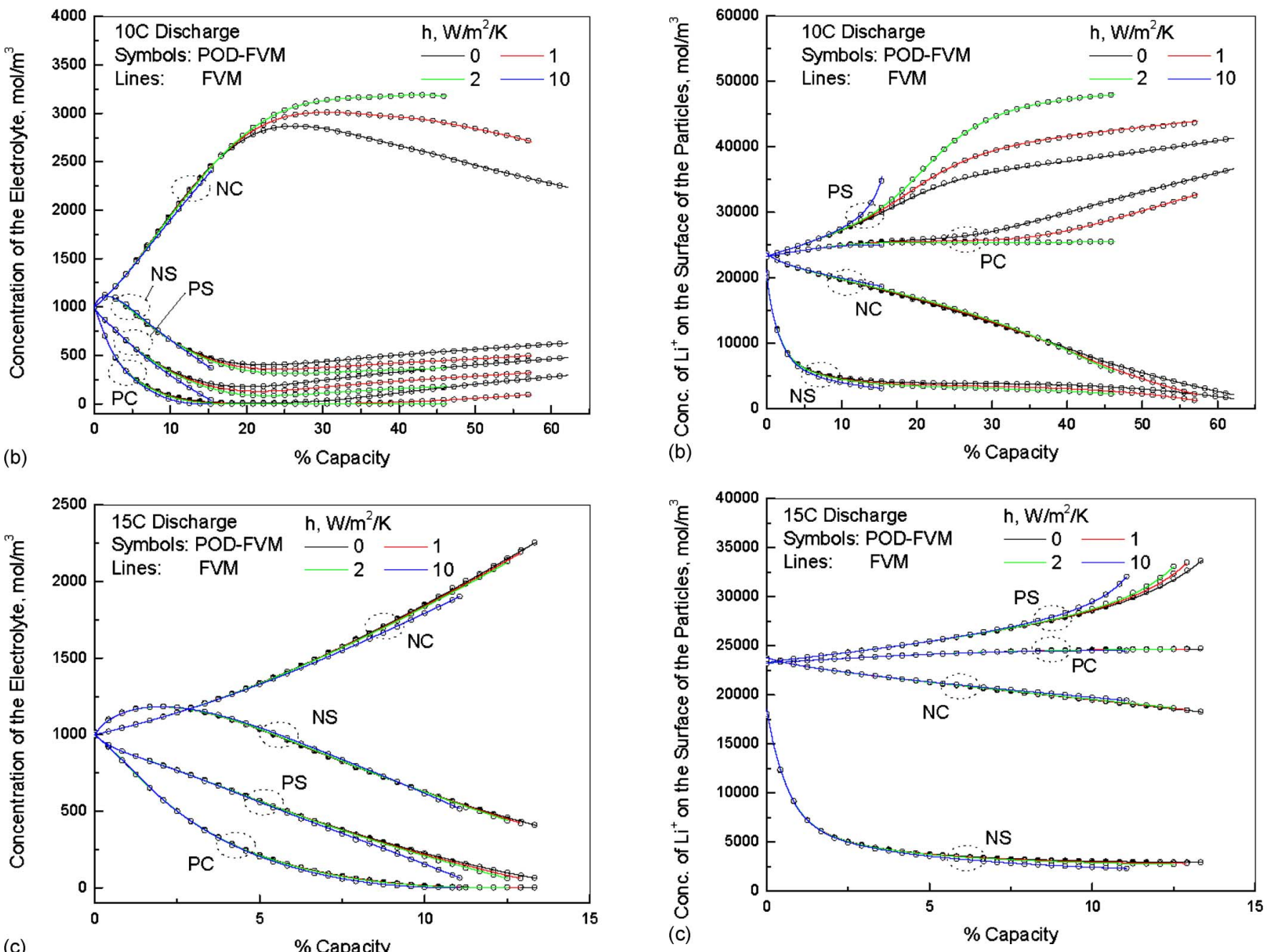

(c)

Figure 8. (Color online) Comparison of the concentrations of the electrolyte at the four interfaces (PC, PS, NS, and $\mathrm{NC}$ ) during the discharge process with the different $\mathrm{C}$ rates $1 \mathrm{C}, 10 \mathrm{C}$, and $15 \mathrm{C}$, which are shown in Fig. 8a-c, respectively. The effective heat transfer coefficient is selected as $0,1,2$, and $10 \mathrm{~W} / \mathrm{m}^{2} / \mathrm{K}$. The symbols are the results of the ROM, and the lines are the results of the rigorous model.

phase, and the high $\mathrm{C}$ rate discharge process is controlled by the mass transfer in the electrolyte. The thermal effect plays an important role on the discharge behavior in the cases (i.e., the 10C dis- charge process) in which the limiting step changes from the mass transport in the solid phase to the mass transfer in the electrolyte. 


\section{Acknowledgments}

The authors gratefully acknowledge the funding support provided by QUALLION LLC under the MDA project STTR Phase II (contract no. HQ0006-09-C-7074): Rechargeable Lithium-Ion Battery Operating Life Model.

University of South Carolina assisted in meeting the publication costs of this article.

\section{List of Symbols}

$c_{\mathrm{e}}$ concentration of the electrolyte, $\mathrm{mol} / \mathrm{m}^{3}$

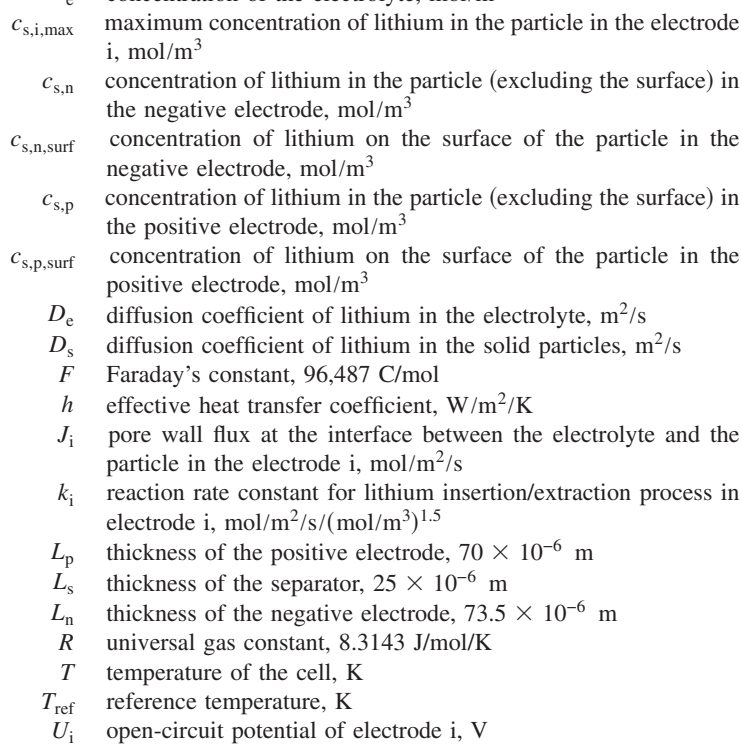

$U_{\mathrm{i}, \text { ref }}$ open-circuit potential of electrode $\mathrm{i}$ at the reference temperature, $\mathrm{V}$

Subscript

$$
\begin{array}{ll}
n & \text { negative electrode } \\
p & \text { positive electrode } \\
s & \text { separator }
\end{array}
$$

Greek

$\eta_{\mathrm{i}} \quad$ overpotential in the electrode $\mathrm{i}, \mathrm{V}$

$\kappa \quad$ ionic conductivity of the electrolyte, $\mathrm{S} / \mathrm{m}$

$\lambda_{i} \quad$ average thermal conductivity of component $i, W / m / K$

$\phi_{1, \mathrm{n}}$ potential of the negative electrode, $\mathrm{V}$

$\phi_{1, p}$ potential of the positive electrode, $\mathrm{V}$

$\phi_{2}$ potential of the electrolyte, $\mathrm{V}$

\section{References}

1. R. Pollard and J. Newman, J. Electrochem. Soc., 128, 491 (1981).

2. D. Bernardi, E. Pawlikowski, and J. Newman, J. Electrochem. Soc., 132, 5 (1985).

3. C. R. Pals and J. Newman, J. Electrochem. Soc., 142, 3274 (1995).

4. C. R. Pals and J. Newman, J. Electrochem. Soc., 142, 3282 (1995).

5. Y. Chen and J. W. Evans, J. Electrochem. Soc., 140, 1833 (1993).

6. Y. Chen and J. W. Evans, J. Electrochem. Soc., 141, 2947 (1994).

7. M. Doyle, T. F. Fuller, and J. Newman, J. Electrochem. Soc., 140, 1526 (1993).

8. T. F. Fuller, M. Doyle, and J. Newman, J. Electrochem. Soc., 141, 1 (1994).

9. W. B. Gu and C. Y. Wang, in Lithium Batteries, S. Surampudi, R. A. Marsh, Z Ogumi, and J. Prakash, Editors, PV 99-25, p. 748, The Electrochemical Society Proceedings Series, Pennington, NJ (2000)

10. K. Kumaresan, G. Sikha, and R. E. White, J. Electrochem. Soc., 155, A164 (2008).

11. V. R. Subramanian, V. Boovaragavan, V. Ramadesigan, and M. Arabandi, J. Electrochem. Soc., 156, A260 (2009).

12. K. Smith and C. Y. Wang, J. Power Sources, 160, 662 (2006).

13. K. Smith, and C.-Y. Wang, Int. J. Energy Res., 34, 556 (2010).

14. L. Cai and R. E. White, J. Electrochem. Soc., 156, A154 (2009).

15. L. Sirovich, Q. Appl. Math., 45, 519 (1987).

16. L. O. Valoen and J. N. Reimers, J. Electrochem. Soc., 152, A882 (2005).

17. G. Berkooz, P. Holmes, and J. L. Lumley, Annu. Rev. Fluid Mech., 25, 539 (1993).

18. F. Riesz and B. Sz.-Nagy, Functional Analysis, Ungar, NY (1955).

19. K. Kunisch and S. Volkwein, J. Optim. Theory Appl., 102, 345 (1999). 